\title{
Ambiguity Analysis for Pulse Compression Radar Using Gold Code Sequences
}

\author{
Todd B. Hale, Michael A. Temple, and Benjamin L. Crossley \\ Air Force Institute of Technology \\ 2950 P Street \\ Wright-Patterson AFB, OH 45433
}

\begin{abstract}
This paper presents analytic, simulation, and measured results of using Gold sequences for radar pulse compression coding. Gold coded waveform performance is characterized using the ambiguity function diagram, synonymous with matched filtering performance. Results indicate Gold coded waveforms offer significant improvement in radar clutter suppression, resolution, and unambiguous range properties.
\end{abstract}

\section{INTRODUCTION}

Pulse compression radar waveforms offer several advantages over uncompressed waveforms. First, a significant increase in unambiguous range can be obtained within transmit power limitations. Second, range and Doppler resolution can be greatly improved. The idea of pulse compression is not new and formally came about from Woodward's work [1] in 1953. His radar waveform analysis and mathematical framework allowed the realization that range resolution is a function of bandwidth rather than pulse width.

Initial pulse compression attempts were realized through linear frequency modulation (LFM) techniques as early as 1940 [2]. As these frequency modulation methods advanced, chirp diverse waveforms rose to the forefront as being the most practical method available. Unfortunately, range recurrent lobe levels degrade (increase) as the number of distinctly coded pulses increases for a constant compression ratio [3]. This degradation prompts research into other pulse compression methods, in particular maximal length (ML) pseudo-noise (PN) sequences and Gold codes, a subset of ML PN sequences. These methods offer manageable cross-correlation properties, i.e., range recurrent lobe characteristics are consistent for all codes within a family. Furthermore, there is no degradation as the number of distinctly coded pulses is increased for constant compression ratio; performance actually improves.

The use of binary sequences for pulse compression essentially began with the introduction of binary phase coding to the radar pulse. The concept is not new, being introduced as early as the 1960s [2,4-6]. Barker codes are probably the most recognized in the radar commu- nity, however their number and length is severely limited with the longest being 13 bits. For this reason, ML PN sequences offer significant practical advantages and are addressed in practically every text dealing with pulse compression $[2,4-6]$. However, these texts offer limited discussion of PN sequence applications and minimal results with the exception of [2], which offers a small representative sketch of an ambiguity surface taken from [7]. The primary disadvantage of PN sequences is their limited number for a particular code length, i.e., compression ratio. For this reason, Gold codes represent a suitable alternative given their increased family size.

This work contributes to previous work through the analytic formulation and accurate characterization of three dimensional ambiguity surfaces for Gold code sequences. Furthermore, a FFT interpretation of the time-frequency autocorrelation function is introduced as a means to speed processing time.

\section{AMBIguity FUNCTION}

The time-frequency autocorrelation function for a unit energy ${ }^{1}$, complex signal $\vec{s}(t)$, or TFACF, is defined as $[1$, $2,6]$

$$
\Phi\left(\tau, f_{D}\right)=\int_{-\infty}^{\infty} \bar{s}^{*}(t-\tau) \bar{s}(t) e^{j 2 \pi f_{D} t} d t,
$$

where $\tau$ represents range induced time delay and $f_{D}$ is the Doppler frequency shift. This mathematical construct represents the matched filter output for any range delayed, Doppler frequency shifted return. The ambiguity function is the magnitude squared of the TFACF,

$$
\chi\left(\tau, f_{D}\right)=\left|\Phi\left(\tau, f_{D}\right)\right|^{2} .
$$

\section{DFT INTERPRETATION}

Equation (1) is best interpreted as a continuous time, continuous frequency Fourier Transform (FT). In this light, the TFACF becomes the Fourier Transform pair

$$
\Phi\left(\tau,-f_{D}\right) \stackrel{F T}{\Longleftrightarrow} \phi(\tau, t),
$$

${ }^{1}$ The ${ }^{-}$in $\bar{s}(t)$ denotes unit energy. 
where $-f_{D}$ is introduced to maintain consistent sign convention. Obviously, the function $\phi(\tau, t)=\tilde{s}(t-\tau) \vec{s}^{*}(t)$ is complicated for the pulse compression techniques we wish to employ and are unlikely to be found in a FT lookup table. Thus, we introduce the relationship between the Discrete Fourier Transform (DFT) and FT as approximated by

$$
\hat{\Phi}\left(\tau,-m f_{s}\right)=t_{s} \sum_{k=0}^{M-1} \phi\left(\tau, k t_{s}\right)\left(e^{j 2 \pi / M}\right)^{-m k},
$$

where $f_{s}$ is the sampling frequency, $t_{s}$ is $1 / f_{s}$, and $M$ is the total number of samples.

For this approximation, one must account for the fact that the DFT assumes a periodic time domain signal. Thus, the spectrum aliases at the fold-over frequency $\left(f_{s} / 2\right)$ and represents the continuous $\mathrm{FT}$ of the original signal only within this range. Ideally, the signal $\phi(\tau, t)$ is bandlimited ensuring the information in the range of Doppler frequencies $-f_{s} / 2, \ldots, f_{s} / 2$ is uncorrupted. We assume $\phi(\tau, t)$ is approximately bandlimited for this work. Comparison of simulation and analytical results show aliasing is evidenced primarily in Doppler sidelobes near the foldover frequency; the impact is insignificant for this work.

\section{Gold Code Sequences}

Pseudo-noise (PN), or pseudo-random, sequences are exactly as their name implies. They represent deterministic sequences that appear to possess properties of random noise. Hence, to an unintended observer they appear truly random. Research of these sequences is primarily motivated for use in spread spectrum communication systems $[8,9]$. Gold code sequences are a special class of $M L$ PN sequences and offer well controlled cross-correlation properties, essentially manageable interference between successive pulses when used for pulse compression radars. Gold codes offer more sequences for a given code length $L$, in fact there are $L+2$ sequences within each family of codes [9]. The cross correlation properties allow the range recurrent sidelobes of the ambiguity function to remain at relatively consistent levels for Gold Codes, regardless of the number $N$ of codes used, i.e., number of pulses distinctly coded. In fact, range recurrent lobe levels actually descrease as $N$ increases. Conversely, chirp diverse or LFM techniques experience a degradation in these sidelobe levels [3] as $N$ increases; the chirp slope lessens on each pulse to accommodate the increased number of coded pulses within a constrained pulse compression ratio, or time-bandwidth product. This decreasing chirp slope reduces the orthogonality of the coded pulses. Gold codes were originally introduced into spread spectrum systems to provide multi-user capability with smaller code lengths, a link back to the limited number of PN sequences for a particular length.
This work characterizes performance for pulse compression radars using two distinct Gold code sequences. These sequences were generated using the $45_{8}$ and $75_{8}$ polynomials from [9] and represent the shortest $\mathrm{ML}$ sequences available for producing Gold code sequences. Given the code period is 31 , there are 33 Gold codes in the family. The two specific sequences chosen for the pulse compression simulation were,

$$
\begin{aligned}
& \text { Gold Code } 1 \text { : }\lceil 0001101111011010001111110100000\rceil \\
& \text { Gold } \text { Code }_{2} \text { : }\lceil 0101100101100001001000000111010\rceil .
\end{aligned}
$$

The transmit waveform coded with these sequences used no phase shift for the 0 s and a phase shift of $\pi$ radians for the 1s (a form of binary phase shift keying).

\section{Results}

The results presented in this section are for a matched filter operating on a pulse train having a pulse repetition interval of $T_{r}=504 \mu \mathrm{sec}$. Each pulse in the train has a width of $T=49.6 \mu \mathrm{sec}$ and contains $31 \mathrm{chips}$, resulting in a chip width of $T_{c}=1.6 \mu \mathrm{sec}$. The analog signal $s(t)$ is digitized in the MATLAB ${ }^{\circledR}$ simulation using four samples per chip for a sample rate of $T_{s}=400 \mathrm{nsec}$, equivalent to $f_{s}=2.5 \mathrm{MHz}$. We chose these parameter values to illustrate concepts involved and represent the medium pulse repetition frequency (PRF) mode of the radar used in the Multi-Channel Airborne Radar Measurement (MCARM.) program [10]. The actual MCARM radar pulse width is $50 \mu \mathrm{sec} ; 49.6 \mu \mathrm{sec}$ was used to ensure the chip width is an integer multiple of the test radar sample rate of $20 \mathrm{MHz}$.

For the $N=2$ pulse case, the ambiguity performance of Gold code sequences is characterized using the surface plot of Fig. 1. The first pulse is phase coded with Gold Code 1 and the second with Gold Code 2 . There are two range recurrent lobes $\left(\tau= \pm T_{r}= \pm 504 \mu \mathrm{sec}\right)$ plus the central primary lobe.

Fig. 2 is an expanded view of the $N=2$ pulse ambiguity surface for $f_{D}$ from $0 \mathrm{~Hz}$ to a maximum of $-1.25 \mathrm{MHz}$. The figure shows the main range lobe centered at $\tau=0$ and the first and second multiples of $f_{c}$, the chip frequency $(625 \mathrm{kHz})$; the second multiple is reduced in amplitude and actually consists of four peaks.

A property of ML sequences, and hence the Gold code subclass, is that they always contain a 010 or 101 bit combination. Hence, the sequences appear to have a (chip) frequency $f_{c}$ equaling the inverse of the chip period $T_{c}$. This waveform periodicity results in small lobes occurring in $f_{D}$ at multiples of $f_{c}=1 / T_{c}$, shown in Fig. 2 for $f_{c}$ equal $625 \mathrm{kHz}$. The two small peaks at $\pm 625 \mathrm{kHz}$ approach zero at $\tau=0$ because the matched filter and received waveforms directly overlap each other; the matched filter is conjugate matched to the received waveform and all phase coding falls out, eliminating the chip frequency component. 


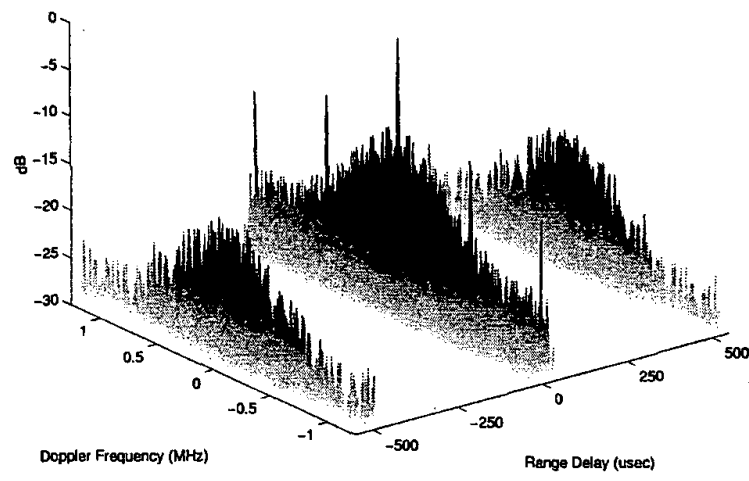

Fig. 1. Ambiguity surface for $N=2$ pulse, Gold coded pulse compression waveform.

There is some concern with aliasing in the previous figures. To avoid aliasing, the waveform $\phi\left(t, f_{D}\right)$ must be bandlimited to the maximum Doppler frequency, e.g., onehalf the sample frequency. We examine (1) for $\tau=0$ to address this issue. Substituting for $\tau$ and using a pulse compression signal, comprised of $N$ pulses and $P$ chips, of the form

$$
s(t)=\sum_{n=0}^{N-1} \sum_{p=0}^{P-1} u_{c}\left(t-n T_{r}-p T_{c}\right) e^{j \psi_{n p}} e^{j 2 \pi f_{o}}
$$

results in

$$
\begin{aligned}
\Phi\left(0, f_{D}\right)= & \frac{T_{c}}{\gamma^{2}} \operatorname{sinc}\left(\pi f_{D} T_{c}\right) \sum_{n=0}^{N-1} \sum_{p=0}^{P-1}\left(\cos \zeta_{n p}\right. \\
& \left.+j \sin \zeta_{n p}\right),
\end{aligned}
$$

where

$$
\zeta_{n p}=2 \pi f_{D}\left\lceil n T_{r}+{ }\left(p+\frac{1}{2}\right)_{T_{c}}\right\rceil
$$

From this we generate an ambiguity surface along $\tau=0$,

$$
\begin{aligned}
\left|\Phi\left(0, f_{D}\right)\right|^{2}= & \frac{T_{\mathrm{c}}^{2}}{\gamma^{4}} \operatorname{sinc}^{2}\left(\pi \mathrm{f}_{\mathrm{D}} \mathrm{T}_{\mathrm{c}}\right) \\
& \sum_{\substack{n=0 \\
N-1}}^{P-1} \sum_{p=0}^{N-1} \sum_{m=0}^{P-1}\left[\cos \left(\zeta_{n p}-\zeta_{m q}\right)\right. \\
& \left.+j \sin \left(\zeta_{n p}-\zeta_{m q}\right)\right],
\end{aligned}
$$

for $T_{r}$ the pulse repetition interval, $T_{c}$ the chip duration, $\psi_{n p}$ the phase associated with pulse $n$ and chip $p, f_{o}$ the carrier frequency, $u_{c}(t)$ a rectangular unit amplitude pulse of width $T_{c}$ starting at $t=0$, and $\gamma^{2}$ the energy in $s(t)$.

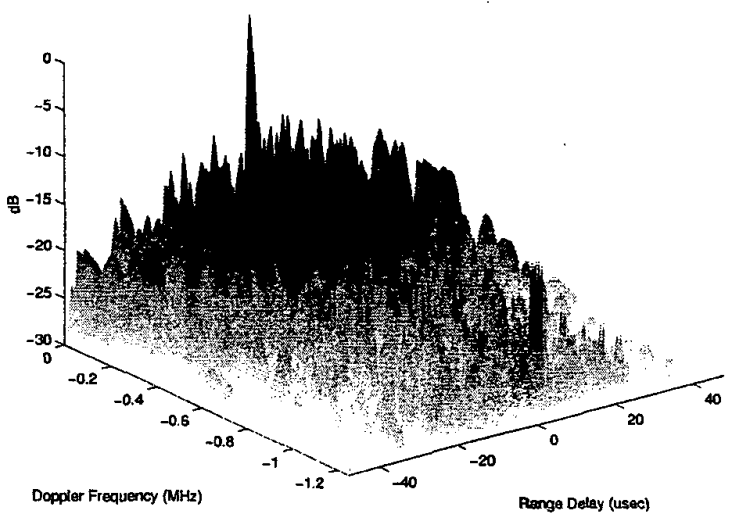

Fig. 2. Expanded view of $N=2$ pulse, Gold coded waveform showing range recurrent lobe structure.

Figs. 3 and 4 show analytic and simulation results, confirming that aliasing is evidenced primarily in the sidelobe regions near the outer extent of $f_{D}$.

Simulation results for the autocorrelation of the two Gold coded waveforms is shown in Fig. 5, essentially a cut of the ambiguity surface for $f_{D}=0$. The autocorrelation function is also equivalent to the matched filter output of a real radar. The autocorrelation function gives an indication of the range resolution capability of the coded waveform.

\section{A. Measured Results}

Measured results were collected using the developmental GP-3 test radar [11]. The radar was originally built under contract for the Air Force Research Laboratory/Sensors Directorate (AFRL/SN) for the purpose of evaluating Space-Time Adaptive Processing (STAP) techniques. AFIT recently acquired the radar and developed an in-house experimental measurement capability. The results presented here serve as a validation for both the radar and computer simulation.

The GP-3 radar measurements were taken under free-space propagation conditions at very low received power levels using a transmit frequency of approximately 9.6 GHz. The GP-3 radar system can simulate practically any waveform provided sample time and sample number constraints are not violated. Basically, the desired transmit waveform is designed using MATLAB ${ }^{(B)}$ on a Unix Workstation and digitally downloaded to the waveform generation and transmission portion of the GP-3 radar, i.e., baseband $\mathrm{D} / \mathrm{A}$ conversion followed by intermediate frequency (IF) conversion and then RF conversion prior to transmission. Upon reception, the signal is downconverted to an IF of $5.0 \mathrm{MHz}(3.5 \mathrm{MHz}$ bandwidth) and then A/D converted for subsequent post-processing. For 


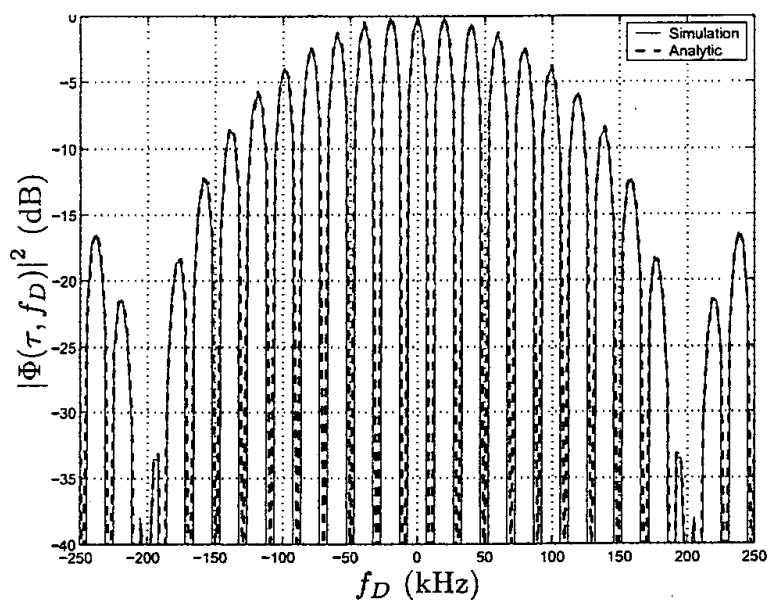

Fig. 3. Analytic and simulation results showing minimal aliasing effects within the main lobe region.

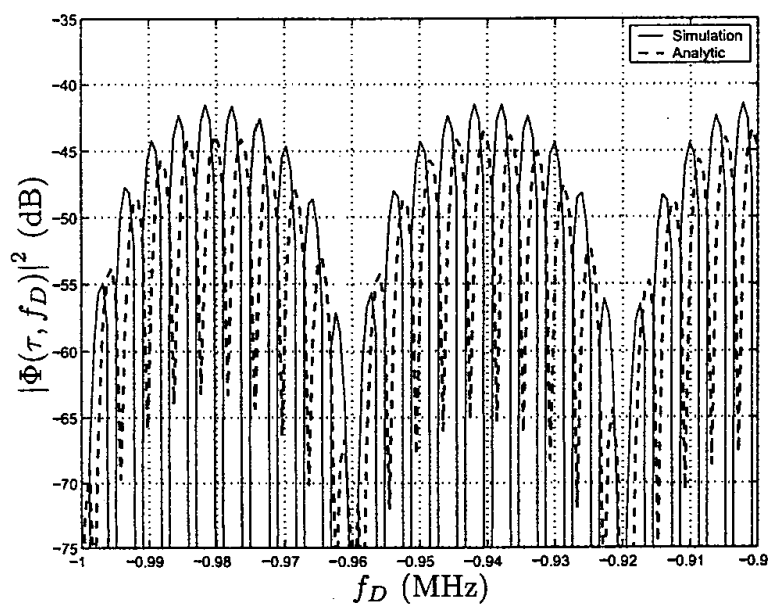

Fig. 4. Analytic and Simulation results showing aliasing effects near outer extent of $f_{D}$.

this effort, all post-processing was done using MATLAB ${ }^{\circledR}$ to generate comparison data. Specifically, post-processing consisted of carrier removal via mixing and low-pass filtering $^{2}$, followed by cross-correlation of the down-converted signal with the transmitted signal envelope, i.e., the baseband transmit signal. This is equivalent to a matched filtering operation and produces results that are directly comparable to the autocorrelation function generated by computer simulation.

Fig. 6 shows the GP-3 post-processed, $N=2$ pulse matched filter output for a signal with an IF signal-to-

\footnotetext{
${ }^{2} \mathrm{~A} 10^{\text {th }}$-order Chebyshev filter with a $0.5 \mathrm{~dB}$ passband ripple and a normalized cut-off frequency of 0.1 (equivalent to $1 \mathrm{MHz}$ ) was used.
}

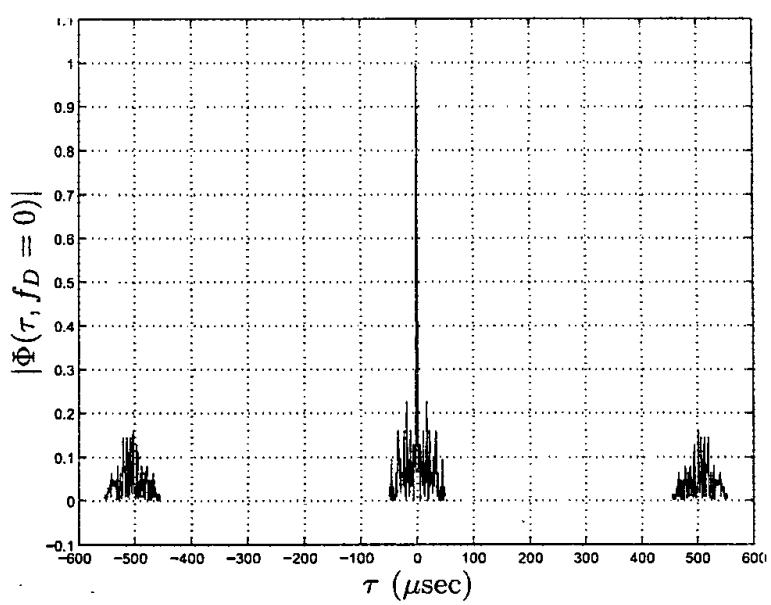

Fig. 5. Simulation results for $N=2$ pulse, Gold coded waveform autocorrelation along $f_{D}=0$ axis.

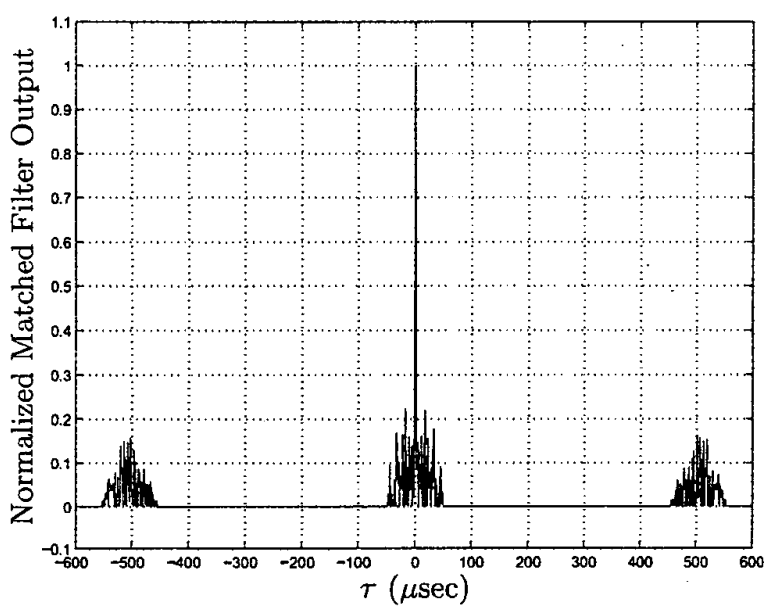

Fig. 6. GP-3 post-processed results for $N=2$ pulse, matched filter output at $\mathrm{SNR}_{\mathrm{IF}}=28.0 \mathrm{~dB}$.

noise ratio $\left(\mathrm{SNR}_{\mathrm{IF}}\right)$ of $28.0 \mathrm{~dB}$. In this case, the GP-3 measured results nearly match the simulation results of Fig. 5 perfectly; these results validate both the computer simulation results and the GP-3 radar performance.

In the interest of evaluating the waveform response to SNR degradation, Fig. 7 is provided for $\mathrm{SNR}_{\mathrm{IF}}-15.0 \mathrm{~dB}$. The $N=2$ pulse Gold coded waveforms appear to be rather robust in terms of signal detectability as SNR degrades.

\section{B. Comparison to Chirp Diverse Waveform}

Chirp diverse waveform coding (multi-slope LFM) offers distinct advantages over PN sequence or Gold coding in some situations. For example, using $N=2$ pulse 


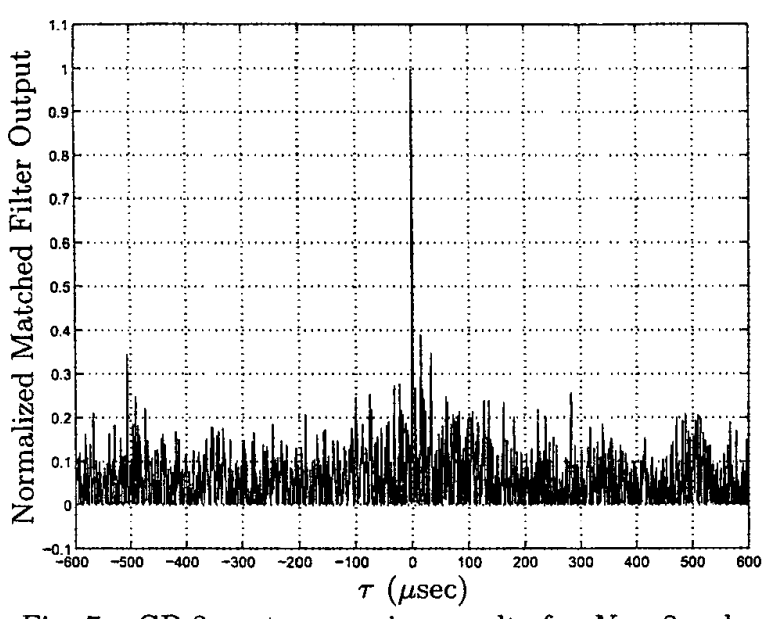

Fig. 7. GP-3 post-processing results for $N=2$ pulse, matched filter output at $\mathrm{SNR}_{\mathrm{IF}}=-15.0 \mathrm{~dB}$ ).

chirp diversity (LFM up and LFM down) provides a drastic performance improvement in autocorrelation function characteristics as shown in Fig. 8. The results here are for a $N=2$ pulse chirped system using slopes of $\pm 625 \mathrm{kHz} / 49.6 \mu$ sec to yield a time-bandwidth product (pulse compression ratio) of 31 , identical to the $N=2$ pulse Gold coded case.

Fig. 9 illustrates the drawback of using chirp diverse waveform coding. As the number of distinctly coded pulses increases, the range recurrent lobe levels increase. This figure illustrates the $\pm T_{r}$ range recurrent lobes for a $N=20$ pulse chirp diverse waveform code. Maximum diversity is used over a total bandwidth of $625 \mathrm{kHz}$ while maintaining a constant pulse compression ratio. As shown, the range recurrent lobes have increased beyond 0.3 . Conversely, the range recurrent lobe levels of the $N=20$ pulse Gold coded waveforms, as shown in Fig. 10 decrease as the number of distinctly coded pulses $N$ increases.

\section{SUMMARY}

As revealed by ambiguity function analysis and supported by GP-3 measured data, Gold code sequences exhibit excellent correlation properties for use in pulse compression waveform coding. Unambiguous range can be greatly reduced and the sequence's low cross-correlation characteristics help keep range recurrent lobe levels to a manageable level. Furthermore, the waveform Doppler response is very good.

The initial concern of the signal waveform $\phi(\tau, t)$ being sufficiently bandlimited to permit use of the DFT approximation did not prove problematic. An analytic solution was developed and confirmed using both simulation and measured radar results. Decreasing sample time $T_{s}$ alle-

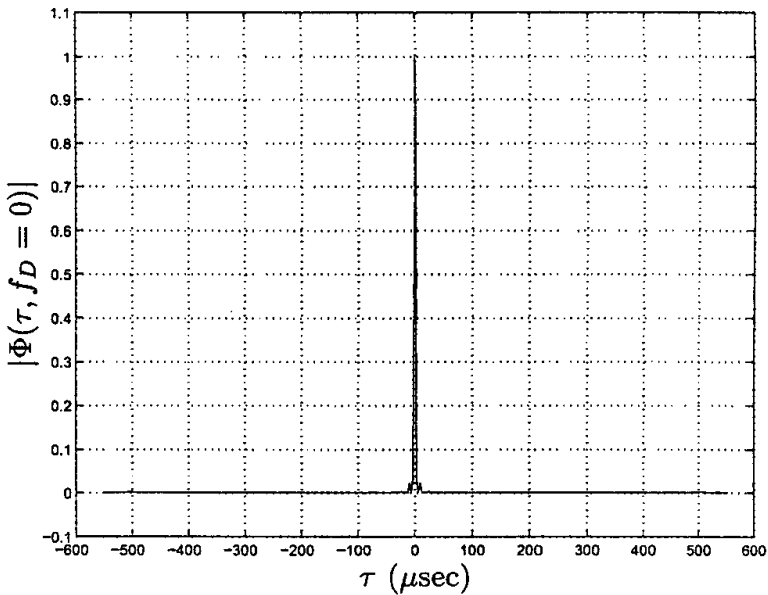

Fig. 8. Simulation results for $N=2$ pulse, chirp diverse waveform autocorrelation function.

viates any aliasing issues. However, there is a trade-off in that computer memory requirements are correspondingly increased.

Of particular importance was the comparison of Gold sequence and chirp diverse waveform coding performance. As implemented, the Gold coded waveforms represented a set of binary, phase-coded waveform possessing nearlyorthogonal correlation characteristics. By comparison, the chirp diverse waveforms set was essentially a series of LFM pulses, each with a distinct chirp slope, possessing poorer correlation characteristics as the number of distinctly coded pulses $N$ is increased. Given identical pulse compression ratios, the Gold coded waveforms generally outperformed the chirp diverse waveform set. This conclusion is based on two factors. First, the Gold code crosscorrelation characteristics are well behaved/structured, i.e., the periodic cross-correlation of any two sequences in a family identically yields the same three values [9]. Second, the cross-correlation of chirp diverse waveforms increases as the number of distinctly coded pulses $N$ increases; a direct result of the constant pulse compression ratio constraint.

\section{ACKNOWLEDGMENT}

The authors would like to express their thanks to Mr. Ed Culpepper of the Air Force Research Laboratory/Sensors Directorate (AFRL/SN), Wright-Patterson $\mathrm{AFB}, \mathrm{OH}$, for making the GP-3 radar available. His timely support and dedication made the measured results presented in this paper possible. 


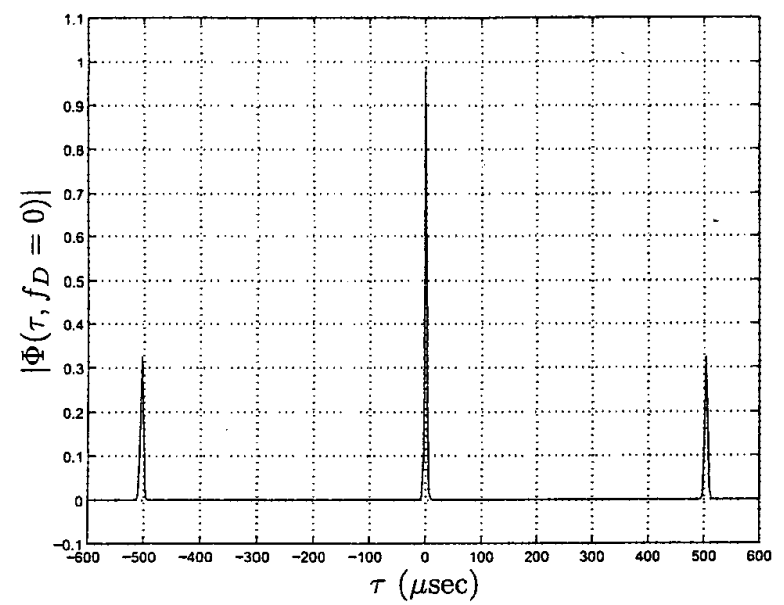

Fig. 9. Simulation results for $N=20$ pulse, chirp diverse waveform autocorrelation function (first two range recurrent lobes at $\pm T_{r}$ shown).

\section{REFERENCES}

[1] P. M. Woodward, ed., Probability and Information Theory, With Applications to Radar. 330 West 42nd Street, New York 36, NY: McGraw-Hill Book Co., Inc., 1953.

2] C. E. Cook and M. Bernfeld, eds., Radar Signals, An Introduction to Theory and Application. Orlando, FL 32887: Academic Press, Inc., 1967.

[3] V. C. Vannicola, T. B. Hale, M. C. Wicks, and P. Antonik, "Ambiguity function analysis for the chirp diverse waveform (cdw)," in Proceedings of the 2000 IEEE International Radar Conference, pp. 666-671, May 2000.

[4] A. W. Rihaczek, ed., Principles of High Resolution Radar. New York, NY: McGraw-Hill, Inc., 1969.

[5] R. S. Berkowitz, ed., Modern Radar, Analysis, Evaluation, and System Design. New York, NY: John Wiley \& Sons, Inc., 1965.

[6] G. V. Morris, ed., Airborne Pulsed Doppler Radar. 685 Canton Street, Norwood, MA 02062: Artech House, Inc., 1988.

[7] T. Sakamoto, Y. Taki, H. Miyakawa, H. Kobayashi, and T. Kanda, "Coded pulse radar system," Journal of Faculty of Engineering at University of Tokyo, no. 27, pp. 119-181, 1964. Unable to obtain and referenced through Cook \& Bernfeld.

[8] B. Sklar, Digital Communications Fundamentals and Applications. Englewood Cliffs, NJ 07632: PrenticeHall, Inc., 1988.

[9] R. L. Peterson, R. E. Ziemer, and D. E. Borth, Introduction to Spread Spectrum Communications. Englewood Cliffs, NJ 07632: Prentice-Hall, Inc., 1995.

[10] D. Sloper, D. Fenner, J. Arntz, and E. Fogle, "Multi-

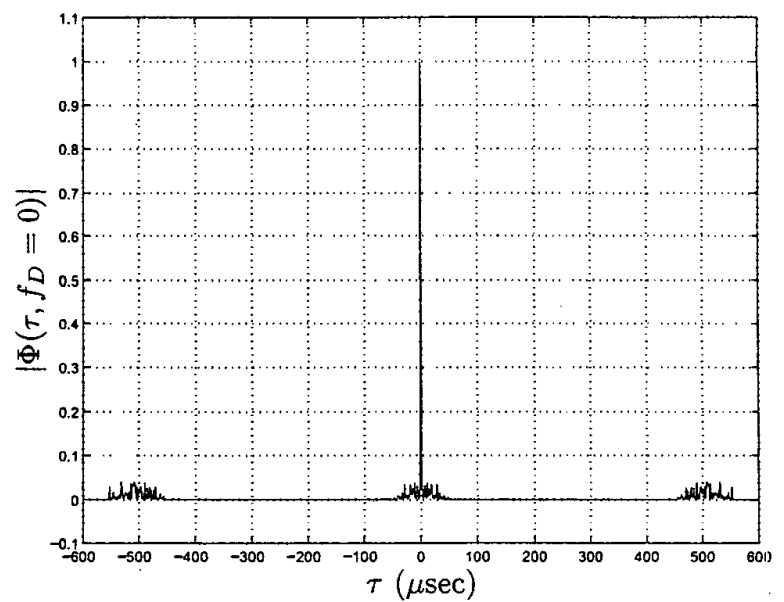

Fig. 10. Simulation results for $N=20$ pulse, Gold coded waveform autocorrelation function (first two range recurrent lobes at $\pm T_{r}$ shown).

channel airborne radar measurement (MCARM), MCARM flight test," Contract F30602-92-C-0161, Westinghouse Electronic Systems, Box 1693, Baltimore, MD 21203, Apr. 1996.

[11] Y. Salama, D. Miedaner, P. Stockmann, and F. Liu, "Gp-3 band signal generation and processing system," Contract AFRL-SN-WP-TR-19991090, Adaptive Technology, Incorporated, 309 Curtis Street, Syracuse, NY 13208, mar 1999.

"The views expressed in this article are those of the author(s) and do not reflect official policy of the United States Air Force, Department of Defense or the U.S. Government." 